\title{
Violation of hyperbolicity via unstable dimension variability in a chain with local hyperbolic chaotic attractors
}

\author{
Pavel V Kuptsov \\ Department of Instrumentation Engineering, Saratov State Technical University, \\ Politekhnicheskaya 77, Saratov 410054, Russia \\ E-mail: p.kuptsov@rambler.ru
}

\begin{abstract}
We consider a chain of oscillators with hyperbolic chaos coupled via diffusion. When the coupling is strong the chain is synchronized and demonstrates hyperbolic chaos so that there is one positive Lyapunov exponent. With the decay of the coupling the second and the third Lyapunov exponents approach zero simultaneously. The second one becomes positive, while the third one remains close to zero. Its finite-time numerical approximation fluctuates changing the sign within a wide range of the coupling parameter. These fluctuations arise due to the unstable dimension variability which is known to be the source for non-hyperbolicity. We provide a detailed study of this transition using the methods of Lyapunov analysis.

PACS numbers: 05.45.Pq, 05.45.Jn, 05.45.-a
\end{abstract}

Keywords: Hyperbolic chaos, Unstable dimension variability, Finite-time Lyapunov exponents, Covariant Lyapunov vectors, Violation of hyperbolicity

Submitted to: J. Phys. A: Math. Gen.

\section{Introduction}

Structural stability is the fundamental property of dynamical systems that implies that qualitative behavior of a system is preserved under perturbations. Structurally stable systems are really significant for theoretical and numerical researches, and especially for practical applications [1, 2]. Simple examples of structurally stable dynamics provide fixed points and limit cycles. The chaotic dynamics is not structurally stable in general; however, a special class of systems with uniform hyperbolicity possesses this property. Mathematical examples of hyperbolic chaotic systems are known since 1960s [3, 4, 5, 6], but physically realizable systems with attractors of this type were discovered sufficiently recently. As reported in [7, 8, [9, 10], simple systems of coupled oscillators exciting alternately possess uniformly hyperbolic attractors of Smale-Williams type. Chaos in 
these systems is related to dynamics of the phases of oscillators in the successive stages of activity governed by a Bernoulli-type expanding circle map.

An attractor is said to be uniformly hyperbolic if the tangent space associated with any of its point can be split into a direct sum decomposition of uniformly expanding and contracting subspaces and this splitting is invariant under the tangent mapping [11]. Tangent vectors from the expanding (contracting) subspace always grow (decay) in time, i.e., corresponding finite-time Lyapunov exponents (FTLEs) are strictly positive (negative). In particular, it means that angles between the expanding and contracting tangent vectors are well separated from zero. The violation of the uniform hyperbolicity can occur due to the emerging of homoclinic tangencies, i.e., tangential intersections of stable and unstable manifolds of the attractor. Trajectories with the exact tangencies are rather untypical in a sense that we almost never hit them choosing random initial conditions. But if they exist, any trajectory can pass infinitely close to them so that the angles between expanding and contracting tangent vectors can be arbitrary small.

A stronger form of non-hyperbolicity is unstable dimension variability (UDV), which is characterized by the coexistence, in the chaotic attractor, of invariant periodic or chaotic orbits with a different number of unstable directions [12, 13]. Since trajectories of the system can pass close to these orbits, the dimension of their unstable and stable manifolds varies. Thus the attractor with UDV does not admit the invariant splitting of the tangent space into strictly expanding and strictly contracting subspaces, so that the conditions of the uniform hyperbolicity fail.

Due to the variations of the unstable manifold dimension, the closest to zero FTLE fluctuates changing its sign [14, 12, 13]. Its distribution spreads both in positive and in negative semi-planes, and the equal probabilities for positive and negative values of the FTLE indicate the strongest UDV [15].

Floating point computations employed in modeling of a dynamics are always subjected to errors caused by finite-precision computer arithmetic. If the dynamics is chaotic, numerical trajectory diverges from the modeled true trajectory. However, if the system holds the shadowing property, there exists a true chaotic trajectory which stays uniformly close to the numerical one for a certain time interval. This shadowing trajectory may not correspond to the initial conditions which was used to compute the numerical trajectory, however its very existence indicates that the computed statistical results are valid. For hyperbolic chaotic systems the time of shadowing is unlimited [16, 17], and most of chaotic systems with homoclinic tangencies also have a reasonably long shadowing time [18]. For systems with UDV the shadowing time can be small which is a serious obstacle for computer modelings [14, 12, 19]. In [14] the estimates for shadowing time and distance are found to be functions of computer round-off error and variance of the sing-changing FTLE. In [15] the scaling law is suggested and the characteristic hyperbolicity exponent is introduced that describe the growth of errors of trajectory averages for systems with the sign-changing FTLE. Altogether, real systems with UDV present many challenges for theoretical and experimental investigation [20, 19]. 
One of the typical situation where UDV occurs is the loss of synchronization of identical coupled chaotic systems [13, 21, 22]. As argued in [23] there are two mechanisms for UDV in this case. While the coupling is sufficiently strong so that the overall synchronization attractor is stable, its embedded periodic orbits can lose the transverse stability. Since for different orbits it can happen at different coupling strengths, it constitutes the UDV mechanism of the first type. In this case a trajectory, started outside but close to the synchronization attractor, quietly approaches it for some time, but then it can pass near a transversally unstable orbit that results in chaotic burst. This behavior, referred to as on-off intermittency [24, 25], is the one of observable manifestations of UDV. The embedded unstable periodic orbits undergoes bifurcations as the coupling decays so that new saddle and repelling orbits appear outside of the synchronization manifold. The set of new orbits is called the emergent set [23]. Beyond the blowout bifurcation [26] where the synchronization attractor becomes transversally repelling, the most of the emergent set is incorporated into the new non-synchronous attractor. Since the orbits from this set can have different dimensions of their unstable manifolds, the UDV occurs, and this is the second mechanism.

Recently the interest to high-dimensional or spatially extended hyperbolic systems has been renewed after the discovery of the numerical algorithms for covariant Lyapunov vectors $(\mathrm{CLVs})$ (see [27, 28] for the original papers, and [29, 30] for reviews). One way to obtain such a system is to build it by coupling together low-dimensional hyperbolic chaotic subsystems. Examples are coupled map lattices with hyperbolic chaos considered in [31, 32] or the diffusive medium with local uniform hyperbolicity suggested in [33]. Less straightforward approach is to identify or create in an extended system the mechanism of spatial modes interactions resulting in hyperbolic chaos. This approach is implemented in 34 for Turing patterns.

Previously, in paper [33] we considered the diffusive medium with local hyperbolic attractor and with no-flux boundary conditions. When spatial coupling is strong, the medium is synchronized and demonstrates uniformly hyperbolic chaos. This regime is characterized by the single positive Lyapunov exponent. Decreasing the coupling results in the desynchronization accompanied by the emerging of the second positive Lyapunov exponent. However, the hyperbolicity survives, so that the system demonstrates a kind of hyperbolic hyper-chaos. The violation of the hyperbolicity occurs only when the third Lyapunov exponent becomes positive.

In this paper we study a system which is very similar to that one presented in [33]. Namely, this is a chain of oscillators with hyperbolic chaos of Smale-Williams type coupled diffusively. The major difference is that the boundary conditions are now periodic. This results in the degeneration of the Lyapunov spectrum so that the second and the third exponents coincide in the synchronous regime. Decreasing the coupling results in their simultaneous approaching to zero where the synchronization is broken. Above the desynchronization threshold the second Lyapunov exponent grows while the third one stays near zero. Numerical, i.e., finite time, approximation of this Lyapunov exponent largely fluctuates frequently changing its sign, and this happens for a wide 
range of coupling strengths. This behaviour is a result of UDV. Subsequent decay of the coupling strength results in the growth of the third Lyapunov exponent and thus to the ceasing of UDV.

The desynchronization of a chain of chaotic elements via UDV is studied in [35] for the lattice of coupled logistic maps. The coupling is varied from the nearest neighborhood to the global coupling. The basic phenomenology related to this system is revealed and discussed, such as UDV, on-off intermittency, FTLE fluctuations and shadowing. In our investigation below, instead of maps we consider a chain of oscillators that are uniformly hyperbolic and admit physical implementation [8]. Moreover, we concentrate on UDV effect occurring beyond the blowout bifurcation due to unstable invariant orbits coexisting with the non-synchronous attractor.

The paper is organized as follows. In section 1 the local oscillators with hyperbolic chaos are discussed, section 2 introduces the system under consideration, and in section 3 we consider the details of the transition to the non-hyperbolic chaos. Namely, we show how the distance to the synchronization manifolds grows as the coupling strength decays, section 3.1; analyze Lyapunov exponents as well as FTLEs, sections 3.2 and 3.3. respectively; then we compute angles between tangent subspaces, section 3.4. finally, discuss the structure of CLVs, section 3.5. Section 4 summarizes the results.

\section{Local oscillators with hyperbolic chaos}

The building block for our model is a set of amplitude equations for the system of two coupled van der Pol oscillators with alternating excitation that is known to demonstrate uniformly hyperbolic chaos [7, 10, 9, 36]:

$$
\begin{aligned}
& \dot{a}=A a \cos (2 \pi t / T)-|a|^{2} a-\mathrm{i} \epsilon b, \\
& \dot{b}=-A b \cos (2 \pi t / T)-|b|^{2} b-\mathrm{i} \epsilon a^{2} .
\end{aligned}
$$

Here $a$ and $b$ are complex dynamical variables, $A$ controls the magnitude of the excitation, $\epsilon$ is the coupling parameter and $T$ is the period of excitation. The subsystems are excited in the alternating manner. The period of the excitation $T$ is assumed to be large with respect to the duration of transient processes in the subsystems controlled by $A$. Suppose at some instant the first oscillator is excited and its amplitude $|a|$ is high. Hence, the second one is suppressed so that its amplitude $|b|$ is small. The coefficients in (1) are real except for the coupling. It means that the phases can be changed only as a result of the interaction between the subsystems. But when $a$ is excited, $|b|$ is small, and its action on $a$ is negligible. Thus, the phase of $a$ remains constant during the excitation stage. The backward influence from $a$ to $b$ is high, and the coupling term is proportional to $a^{2}$. It means that after the interval $T / 2$ the oscillator $b$ at the onset of its own excitation inherits a doubled phase of $a$ (the phase also gets a shift $-\pi / 2$ because of the imaginary unit at the coupling term). Now the roles of the subsystems are exchanged. The phase of $b$ remains constant when this subsystem is excited, and at the end, after the other lapse $T / 2$, the phase is returned back to $a$ through a linear 
coupling term (also with the shift $-\pi / 2$ ). As a result, the first oscillator $a$ doubles its phase during the period $T$.

The above discussion allows us to write down a stroboscopic map for a series of phases $\phi_{n}=\arg a(n T)$ that are measured after each period $T: \phi_{n+1}=2 \phi_{n}-\pi \bmod 2 \pi$. This map demonstrates uniform hyperbolic chaos: the rate of exponential divergence of two close trajectories is identical in each point of the phase space and equal to $\ln 2$. Since the described mechanism is the solely responsible for the chaos in the system (11), one can expect that the system itself demonstrates hyperbolic chaos. The detailed analysis presented in [9, ?] confirms that the stroboscopic map, whose variables coincide with $a(t)$ and $b(t)$ at $t=t_{n}=n T$ is indeed hyperbolic. In particular, the largest Lyapunov exponent of this map is very close to $\Lambda_{1}=\ln 2$.

\section{The model system}

First, using the oscillators (11) as local elements, we construct an active diffusion medium studied in [33]:

$$
\begin{aligned}
& \partial_{t} a=A \cos (2 \pi t / T) a-|a|^{2} a-\mathrm{i} \epsilon b+\partial_{x}^{2} a, \\
& \partial_{t} b=-A \cos (2 \pi t / T) b-|b|^{2} b-\mathrm{i} \epsilon a^{2}+\partial_{x}^{2} b .
\end{aligned}
$$

Since we are going to solve these equations numerically, the discretization of spatial derivatives can be taken into account explicitly. Thus we obtain a chain of $N$ hyperbolic oscillators with the diffusive coupling:

$$
\begin{aligned}
& \dot{a}_{i}=A \cos (2 \pi t / T) a_{i}-\left|a_{i}\right|^{2} a_{i}-\mathrm{i} \epsilon b_{i}+\kappa\left(a_{i}\right) / h^{2}, \\
& \dot{b}_{i}=-A \cos (2 \pi t / T) b_{i}-\left|b_{i}\right|^{2} b_{i}-\mathrm{i} \epsilon a_{i}^{2}+\kappa\left(b_{i}\right) / h^{2} .
\end{aligned}
$$

Here $\kappa\left(z_{i}\right)=z_{i-1}-2 z_{i}+z_{i+1}, i=0, \ldots N-1$ and $h$ is the step of discretization. The length of the system is $S=N h$, and the diffusion coefficients are rescaled to units. The spatial coupling in this chain is controlled by varying $S$. While for the original continuous model (2) this is an unambiguous manipulation, dealing with numerical simulations we have two main strategies which, in principle, can give different results. The first one is to vary $N$ while keeping constant $h$. In this way the thermodynamic limit can be investigated where the number of degrees of freedom is infinite. The second approach is to vary $h$ at constant $N$ and thus to investigate the continuous limit at $h \rightarrow 0$. One more reasonable approach is to consider the continuous limit at constant $S$ : increasing of $N$ and proportional decreasing of $h$. Below we shall vary $h$ at constant $N$.

Thus, parameter $h$ plays the role of a reciprocal coupling strength, i.e., high values of $h$ correspond to weak coupling and vice versa. The lowest reasonable values of $h$ are those for which the chain demonstrates full chaotic synchronization. The highest ones are attained when the oscillators are effectively uncoupled. Each partial oscillator has one positive Lyapunov exponent. As we shall see below, the number of positive Lyapunov exponents for the whole chain grows with $h$. Thus, the chain is effectively uncoupled when the total number of positive exponent is equal to $N$. 
In [33] equations (2) was studied with the no-flux boundary conditions. In the present work we consider the chain (3) with the periodic boundary conditions.

Since the uniform hyperbolicity is proven only for the stroboscopic map corresponding to (1), in what follows we shall represent the results of simulations of (3) in terms of the stroboscopic map whose state variables coincide with the chain variables $a_{i}(t)$ and $b_{i}(t)$ at $t=t_{n}=n T$. In fact, it merely means that the Lyapunov exponents $\lambda_{i}$ are computed first for the continuous time system (3), and then they are multiplied by the time step $T$, i.e., $\Lambda_{i}=T \lambda_{i}$. In the same way FTLEs of (3), computed for time intervals $n T$, are related to FTLEs $L_{i}(n)$ of the stroboscopic map. Finally, the Lyapunov vectors for the stroboscopic map as well as their angles are obtained for the continuous time system at $t=n T$.

\section{Transition from hyperbolic to non-hyperbolic chaos via unstable dimension variability}

\subsection{The distance to the synchronization manifold}

Small $h$ produces strong coupling. In this case the oscillators of (3) are synchronized, and the stroboscopic map for the chain demonstrates hyperbolic chaos with the single positive Lyapunov exponent $\Lambda_{1} \approx \ln 2$. The attractor in the synchronous regime will be referred to as synchronization attractor, and the manifold containing this attractor $a_{i}=a_{j}, b_{i}=b_{j}$, where $0 \leq i, j \leq N-1$, will be referred to as the synchronization manifold.

Increasing $h$ we expect to observe the destroying of the synchronous regime. To characterize the deviation of the system from the synchronization manifold it is convenient to use new variables measured along directions transverse to this manifold, that are introduced in [37]. (Another formulas for transverse directions can be defined, see for example [38].) There are $N-1$ such variables that can be computed as

$$
a_{i}^{\prime}(t)=a_{i}(t)-\frac{1}{i} \sum_{k=0}^{i-1} a_{k}(t),
$$

where $i=1, \ldots N-1$. In the same way they are introduced for $b_{i}(t) \pm$. Now given the transverse coordinates, we can find the distance from the synchronization manifold as

$$
\rho(t)=\sqrt{\sum_{i=1}^{N-1}\left|a_{i}^{\prime}(t)\right|^{2}+\left|b_{i}^{\prime}(t)\right|^{2}}
$$

To compute the distance corresponding to the stroboscopic map we have to take into account variables $a_{i}(t)$ and $b_{i}(t)$ only at $t=n T$.

When $h \leq 1.04$ the distance $\rho(n T)$ vanishes, i.e. the chain is synchronized. We note that the full synchronization of $N$ chaotic elements with the local coupling is possible

$\ddagger$ To obtain a complete set of new variables, one have to add here the longitudinal variables $a_{0}^{\prime}$ and $b_{0}^{\prime}$ computed as averaged $a_{i}$ and $b_{i}$, respectively. 
only when $N$ is finite and below some threshold. Paper [35] shows that given $N$ one can obtain the synchronization increasing the coupling range. In [37] we demonstrate that the number of elements that can be synchronized depends on the largest Lyapunov exponent of the partial element: the higher is the exponent, the smaller is $N$.

Figure 1 shows the time dependence of $\rho(n T)$ above the desynchronization threshold. In Fig. 1(a) we observe alternating quiescent and bursting phases. This regime is called on-off intermittency [24, 39]. It is typical for coupled systems losing chaotic synchronizations. Unstable periodic orbits embedded into the synchronous attractor becomes transversally unstable before the attractor as a whole do so. As a result a trajectory not exactly belonging to the attractor may approach to it for sufficiently long time, but the passings close to points of the unstable orbits result in the burstings. Since the embedded periodic orbits and the attractor as a whole have different dimensions of unstable and stable manifolds, the UDV takes place, which, in particular, results in the violation of the uniform hyperbolicity [12, 13. According to the notation of [23], this is the first mechanism of UDV.

Figure 1(b) is plotted for a larger value of $h$. The intermittency is absent, and irregular oscillations are observed instead. It means that now the synchronous attractor as well as the most of the unstable periodic orbits are transversally unstable so that trajectories never stay close to the synchronization manifold for a long time. Notice that $h$ in this panel is just a little bit higher then in the panel (a). It means that the transverse destabilization of embedded periodic orbits takes place for our system within a very narrow range of the coupling parameter. Previously we already observed the very narrow range of on-off intermittency and riddling for a system of two coupled oscillators (1), see [40]. Also the narrow range of the on-off intermittency regime is reported in 35 for the lattice of coupled logistic maps.

Averaging $\rho(t)$ over a large number of trajectory points results in the characteristic value $\langle\rho\rangle$ indicating how far the system is off the synchronization manifold. Figure 2(a) shows the growth of $\langle\rho\rangle$ as $h$ grows. The transition to non-synchronous regime occurs in the vicinity of $h \approx 1.04$.

\subsection{Lyapunov exponents and the manifestation of $U D V$}

Now we turn to the Lyapunov exponents. Figure 2(b) demonstrates the first six Lyapunov exponents versus the coupling parameter $h$. First of all notice that the largest one remains almost constant, $\Lambda_{1} \approx \ln 2$. In the synchronous regime all other exponents are negative. Due to the symmetry imposed by the periodic boundary conditions, the second and the third Lyapunov exponents coincide, $\Lambda_{2}=\Lambda_{3}$. When $h$ grows these exponents approach zero simultaneously. Then the system leaves the synchronization manifold that results in the breakdown of the degeneracy. The second exponent becomes positive, while the absolute value of the third one remains small.

Figure 3 shows the behavior of the second and the third Lyapunov exponents in a close vicinity of the desynchronization onset. One can see the fluctuations of the 


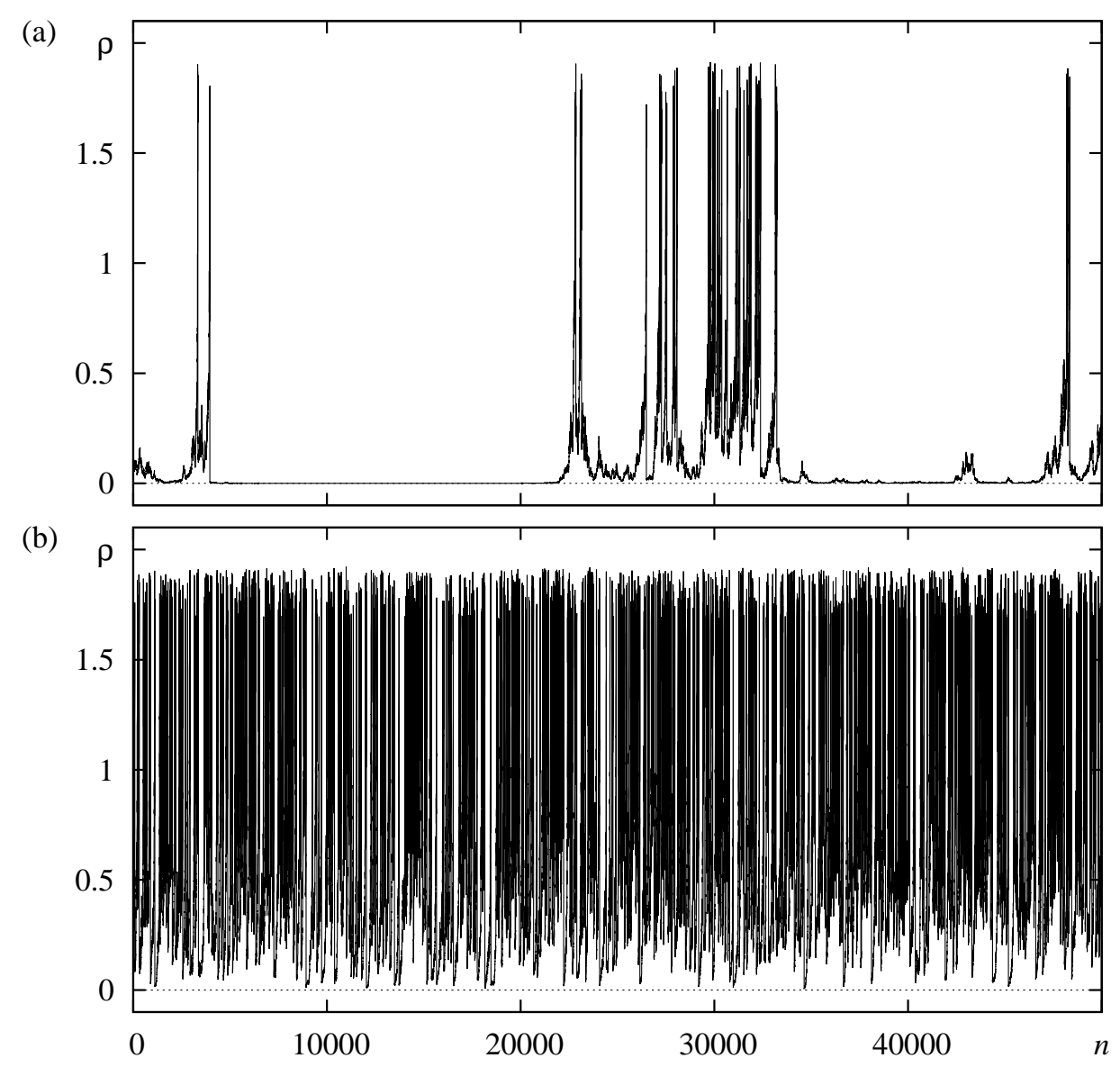

Figure 1. The distance $\rho(n T)$ to the synchronization manifold vs. time step $n$ for (a) $h=1.05$, and (b) $h=1.06$. $N=16, A=3, T=5, \epsilon=0.05$.

numerical approximation of $\Lambda_{2}$, as marked by the dashed vertical lines. The signchanges are very rare, but nevertheless occur. Moreover, here the on-off intermittency is observed, as seen in Fig. 1(a). This agrees with our above discussion of the UDV arising in this area due to the loss of the transverse stability of the unstable periodic orbits embedded into the synchronization attractor.

To the right of the marked area in Fig. 3 the numerical approximation of $\Lambda_{2}$ does not fluctuate anymore. The inset in Fig. 2(b) shows however that $\Lambda_{3}$ changes its sign. Inspecting the results of five independent computations of $\Lambda_{3}(h)$ one can see that the numerical approximation of $\Lambda_{3}$ often passes up and below the zero axis as $h$ grows. The fluctuations occur within a wide range of $h$ and disappear at $h \approx 1.4$ where the exponent leaves the vicinity of zero. These fluctuations indicate the presence of UDV arising here via the second mechanism due to the emergent sets appearing outside the synchronization attractor [23].

Further growth of $h$, i.e., the decay of the coupling strength, results in the vanish of UDV. The third Lyapunov exponent leaves the vicinity of zero in a smooth way so that the probability of its sign change vanishes. Then, as the coupling strength 

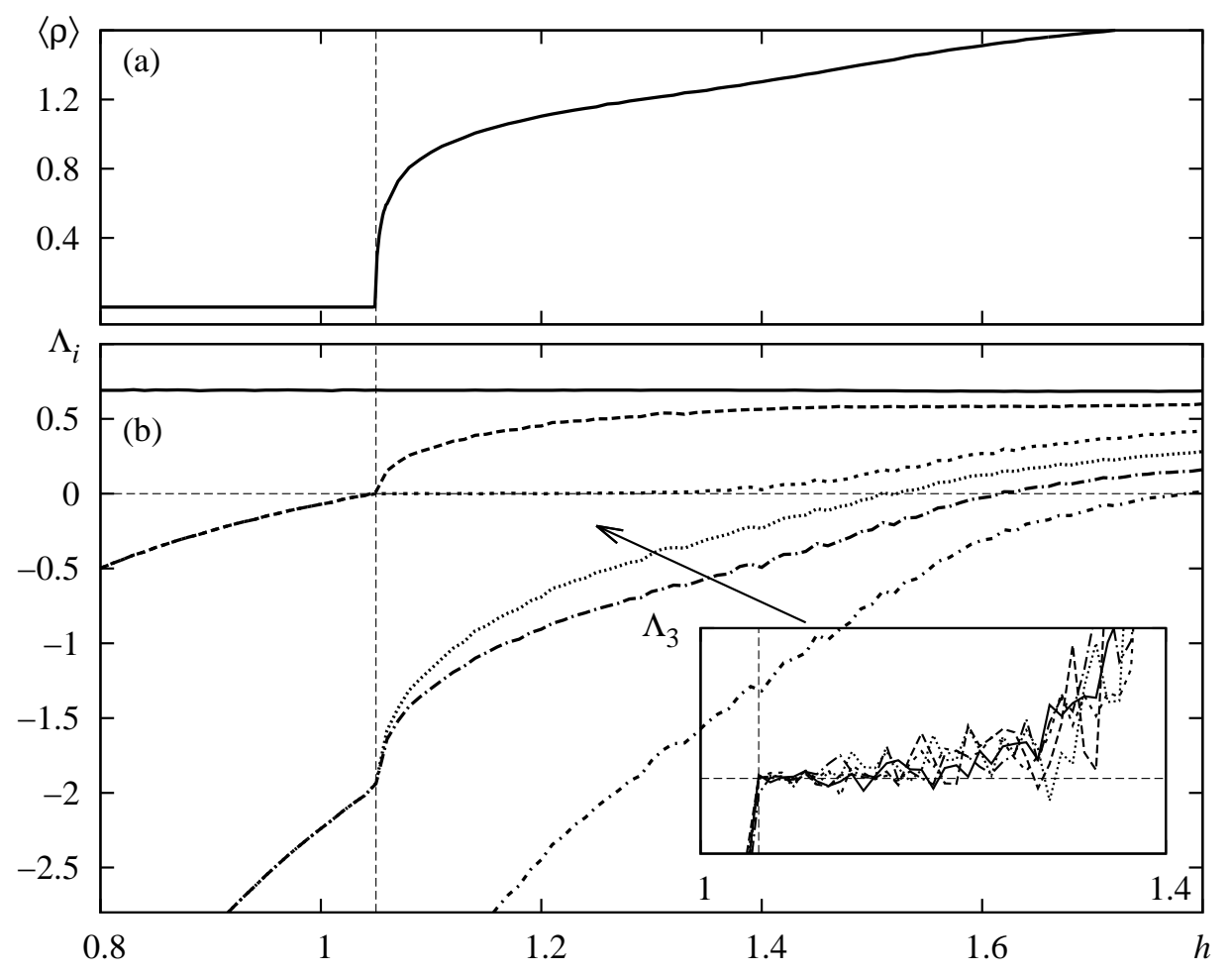

Figure 2. (a) The average distance $\langle\rho\rangle$ form the synchronization manifold and (b) the first six Lyapunov exponents vs. the reciprocal coupling strength $h$. The inset in the panel (b) shows $\Lambda_{3}$ in the larger scale. $N=16, A=3, T=5, \epsilon=0.05$.

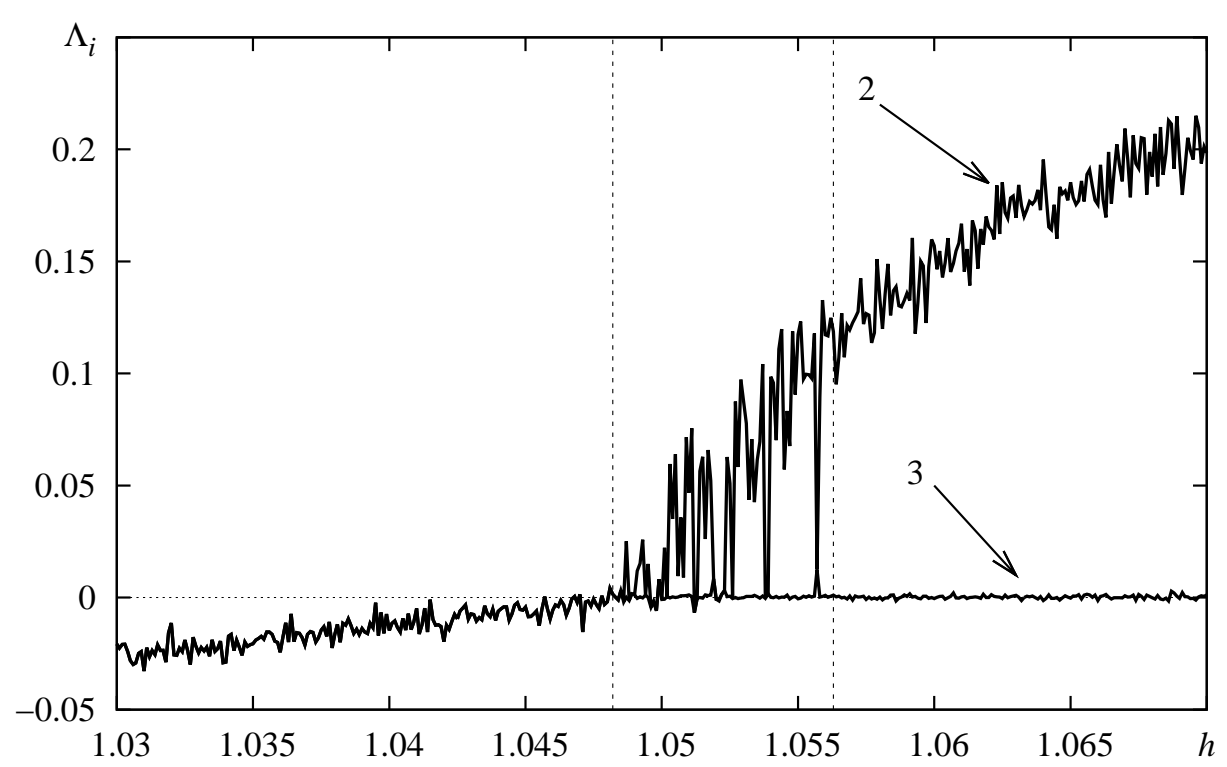

Figure 3. The second and the third Lyapunov exponents vs. $h$ in the area of the desynchronization. Dashed vertical lines mark the range with on-off intermittency.

decays further, the Lyapunov exponents becomes positive one by one, which is typical for spatio-temporal chaotic systems. 


\subsection{Fluctuations of finite-time Lyapunov exponents}

Fluctuations of $\Lambda_{3}$ observed in Fig. 2(b) are obviously explained by the fact that numerically we can obtain only a finite-time approximation of Lyapunov exponents. Thus, it is natural to take these fluctuations into account explicitly, and discuss the FTLEs $L_{i}(n)$, such that

$$
\Lambda_{i}=\lim _{n \rightarrow \infty} L_{i}(n) .
$$

We recall that in fact the FTLEs will be computed for the continuous times system (3) for time interval $n T$.

Figure 4 shows the plot of $L_{3}(1)$ against the distance $\rho$ to the synchronization manifold for $10^{5}$ trajectory points. The panel (a) corresponds to the on-off intermittency case, shown in Fig. 1(a). The bulk of points is located near the synchronization manifold and $L_{3}(1)$ fluctuates there, changing its sign. This again confirms that the first mechanism of UDV dominates in this case, i.e., the UDV appears due to the periodic orbits embedded into the synchronization manifold. Nevertheless, there are rarer fluctuations of high amplitude at high $\rho$. They appear due to the second mechanism, i.e., due to the emergent set of orbits outside the synchronization manifold. The panel (b) also corresponds to the UDV case, but at higher $h$. Now $L_{3}(1)$ at small $\rho$ can only approach zero axis from above without crossing it. It means that all embedded periodic orbits are transversally unstable, and the first UDV mechanism does not work. The UDV occurs solely due to the second mechanism.

Figure 5 shows the distributions of the first four FTLEs $L_{i}(128)$ for the case represented in 4(b). The distributions have a Gaussian form: there are a well defined maxima and rapidly decaying tails. Hence the infinite-time Lyapunov exponents has definite values. The third exponent can take both positive and negative values with almost equal probabilities. The detailed inspection of the distribution reveals that the maximum of the curve is located at 0.00168 which can be taken as an approximate value of $\Lambda_{3}$.

To characterize statistical properties of FTLEs, we use the approach of [41]. Employing the Gaussian approximation for distributions, we compute covariances of all pairs $n L_{i}(n)$ and $n L_{j}(n)$ as functions of $n$, then estimate their linear slopes. These slopes are the diffusion coefficients describing the growth rates of (co)variances of $n L_{i}(n)$. We use them to construct a symmetric diffusion matrix $\mathbf{D}$. In fact, below we shall use only the diagonal and sub-diagonal elements of this matrix. The variance of the distribution of $L_{i}(n)$ is $D_{i i} / n$, where $D_{i i}$ is the $i$-th diagonal element of the matrix. For the distributions shown in Fig. 5 the diffusion coefficients are $D_{11}=0.00461$, $D_{22}=0.430, D_{33}=0.0455, D_{44}=2.05$.

Let $\ell$ be an index of the smallest positive infinite-time Lyapunov exponent, and $\ell+1$ corresponds to the largest negative one. Consider the probability of fluctuation of unstable direction $P\left[L_{\ell}(n) \leq 0 \vee L_{\ell+1}(n) \geq 0\right]$. Since Gaussian bivariate cumulative distribution function has a sufficiently complicated closed form expression [42], we shall 


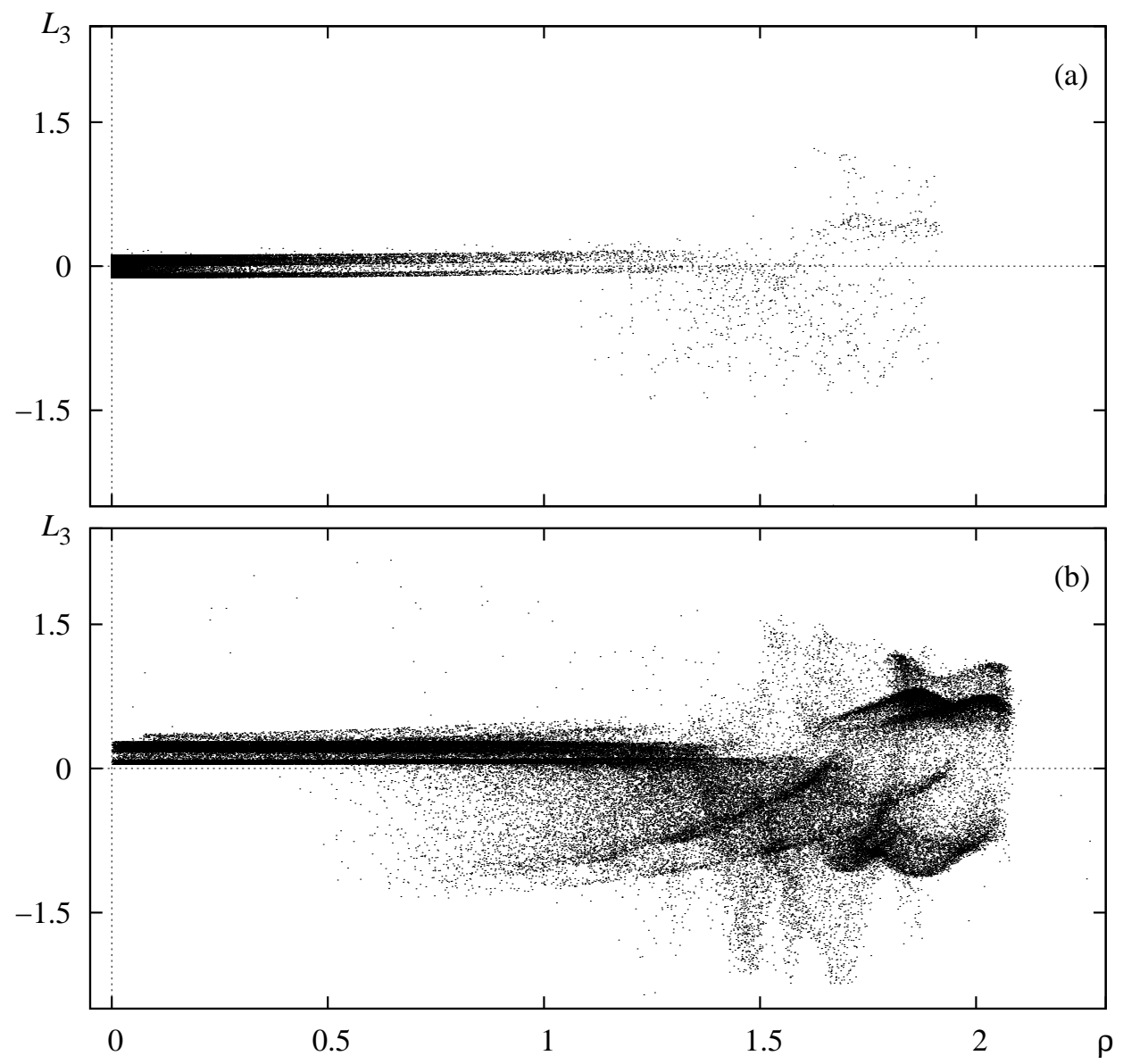

Figure 4. The third FTLE $L_{3}(1)$ vs. transverse distance to the synchronization manifold $\rho$ computed for $10^{5}$ trajectory points. (a) $h=1.05$, (b) $h=1.2$.

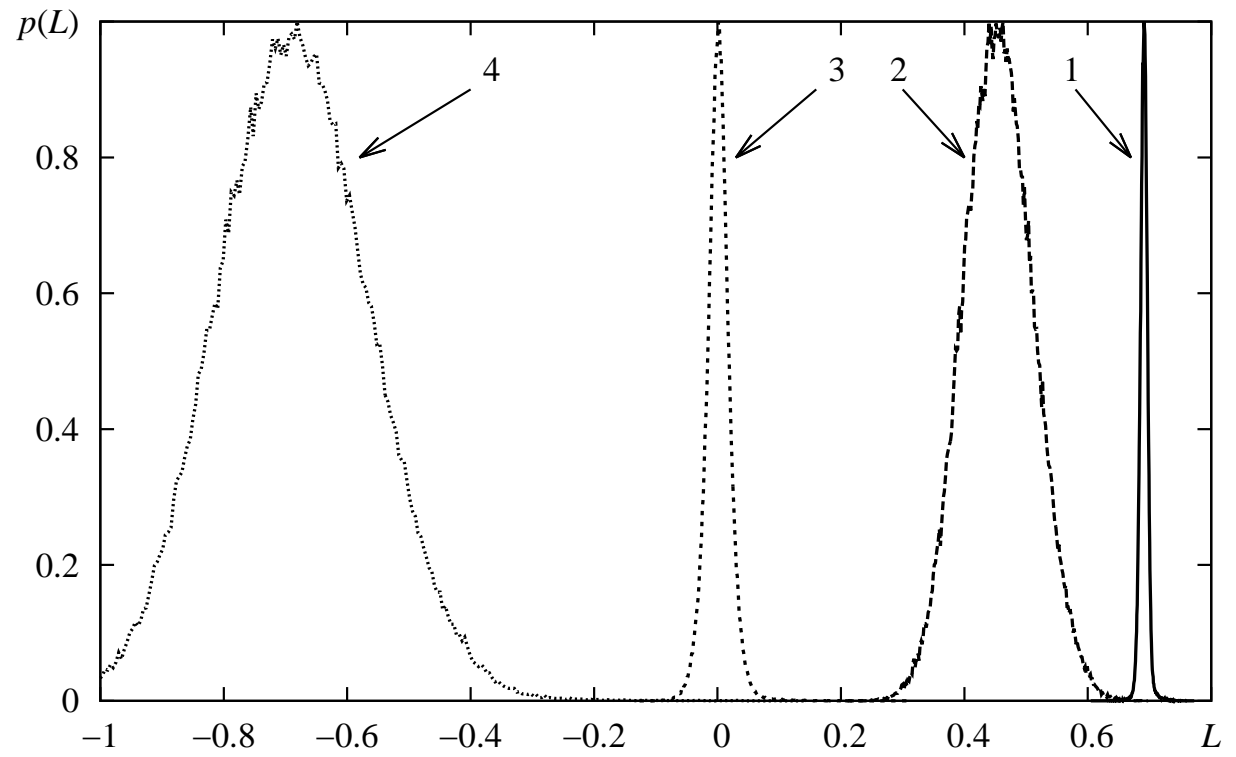

Figure 5. Distributions of the first four FTLEs $L_{i}(128)$ at $h=1.2$. 
Table 1. Closest to zero Lyapunov exponents and fluctuation characteristics of the corresponding FTLEs.

\begin{tabular}{lllllllll}
\hline$h$ & $\ell$ & $\Lambda_{\ell}$ & $\Lambda_{\ell+1}$ & $D_{\ell \ell}$ & $D_{\ell+1, \ell+1}$ & $H_{\ell}$ & $H_{\ell+1}$ & $K_{\ell}$ \\
\hline 0.8 & 1 & 0.690 & -0.499 & 0.00398 & 0.00398 & 7.73 & -5.59 & $\sim 10^{-10}$ \\
1.2 & 3 & 0.00168 & -0.692 & 0.0455 & 2.05 & 0.00558 & -0.342 & 0.428 \\
1.7 & 5 & 0.0897 & -0.105 & 0.646 & 2.03 & 0.0789 & -0.0521 & 0.150 \\
\hline
\end{tabular}

estimate its lower $P_{0}$ and upper $P_{1}$ bounds instead as follows:

$$
\begin{aligned}
& P_{0}<P\left[L_{\ell}(n) \leq 0 \vee L_{\ell+1}(n) \geq 0\right]<P_{1}, \\
& P_{0}=\max \left\{P\left[L_{\ell}(n) \leq 0\right], P\left[L_{\ell+1}(n) \geq 0\right]\right\}, \\
& P_{1}=P\left[L_{\ell}(n) \leq 0\right]+P\left[L_{\ell+1}(n) \geq 0\right], \\
& P\left[L_{i}(n) \leq 0\right]=\operatorname{erfc}\left(\sqrt{n} H_{i}\right) / 2, \\
& P\left[L_{i}(n) \geq 0\right]=\operatorname{erfc}\left(-\sqrt{n} H_{i}\right) / 2,
\end{aligned}
$$

where $H_{i}=\Lambda_{i} / \sqrt{2 D_{i i}}$, and "erfc" stands for the complementary error function. The coefficient $H_{i}$ determines the rate of probability convergence to the asymptotic value as $n$ grows. $\left|H_{i}\right|$ computed for the closest to zero FTLE is related to hyperbolicity exponent $\tilde{h}$, introduced in [15]. In our notation, $\tilde{h}=2\left|\Lambda_{i i}\right| / D_{i i}=\left|H_{i}\right| \sqrt{8 / D_{i i}}$. Both of these coefficients have identical qualitative meaning: their large values indicate fast decay of the FTLE fluctuations with $n$.

Table 1 collects the closest to zero Lyapunov exponents, corresponding diffusion coefficients, and rates of probability convergence for three values of $h$. The first row at $h=0.8$ corresponds to the full synchronization where the oscillations are hyperbolic, the second row at $h=1.2$ represents the case of UDV, and the third row at $h=1.7$ illustrates the situation beyond the UDV where the chaos, as discussed in section 3.4, is nonhyperbolic. Using these data we compute the bounding intervals for unstable manifold dimension fluctuations probability as functions of the averaging length $n$, see Fig. 6. The curves 1 represent the hyperbolic chaos. Observe that the boundaries limiting possible values of the fluctuations probability decay to zero and vanish at $n=0.1$. It means that even at $n=1$ the fluctuations of the unstable manifold dimension are absent. Using the Gaussian approximation we can not detect the strict vanishing of the fluctuations. Nevertheless, the observed rapid decay of the fluctuations probability agrees well with the fact that the system is actually hyperbolic in this case and the fluctuations have to be absent. The curve 3 corresponds to the "common" non-hyperbolic chaos. The fluctuations of the unstable manifold dimension are essential only if we consider FTLEs at small $n$. They decay fast and almost disappear at moderately high $n$. When $n>10^{3}$ the Gaussian approximation predicts negligibly small fluctuations. The UDV case is represented by the curve 2 . The upper boundary of the probability depends on $L_{4}$ that is far from zero and thus does not contribute much to the fluctuations. The fluctuations of $L_{4}$ practically do not influence the unstable manifold dimension variations when 


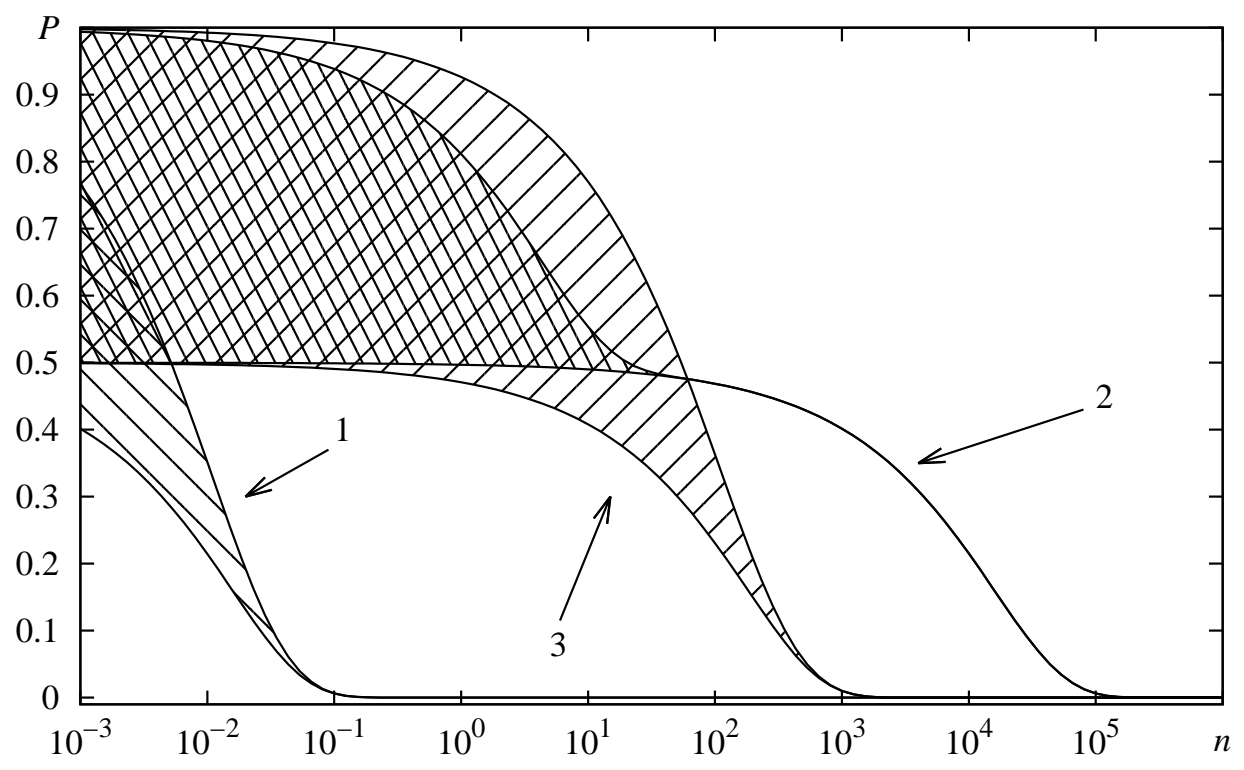

Figure 6. Lower and upper estimations of probability of wrong signs of FTLEs vs. the averaging interval $n$. Curves 1: hyperbolic chaos at $h=0.8$; curves 2 : UDV at $h=1.2$; curves 3 : chaotic dynamics at $h=1.7$.

$n>10^{2}$ where the upper and lower boundaries merge. To the right of this point the unstable manifold dimension fluctuations are basically determined by the crossing zero $L_{3}$. The fluctuations decay very slowly and remain at an essential level up to $n>10^{5}$ that is two order higher then in the "common" case. Notice that the corresponding probability convergence rate $H_{3}$ is one order less than the smallest rate corresponding to the other FTLEs.

Another, rather heuristic, estimate for the magnitude of the unstable dimension fluctuations provide the diffusion coefficients $K_{\ell}=D_{\ell \ell}+D_{\ell+1, \ell+1}-2 D_{\ell, \ell+1}$ that characterise the probability of the negative value of the difference $L_{\ell}(n)-L_{\ell+1}(n)$. As argued in [41, if $\Lambda_{\ell}>\Lambda_{\ell+1}$ and $K_{\ell}=0$ the FTLEs are always ordered correctly, $L_{\ell}(n)>L_{\ell+1}(n)$. Though it does not imply the absence of the unstable dimension fluctuations directly (since $L_{\ell}(n)$ still can be negative), it anyway indicates the low level of "bad" fluctuations of corresponding FTLEs. One can see from table 1 that for the hyperbolic chaos $K_{1}$, as expected, is effectively zero. The "common" chaos is characterized by the moderately small $K_{5}$, and the highest value of $K_{3}$ corresponds to the UDV case.

\subsection{Angles between tangent subspaces}

The tangent space splitting can be introduced using covariant Lyapunov vectors $\vec{\gamma}_{i}(t)$ [29]. Let $\mathcal{A}_{k}$ be a subspace spanned by the first $k$ CLVs, $\mathcal{A}_{k}=\operatorname{span}\left\{\vec{\gamma}_{i}(t) \mid i=\right.$ $1, \ldots, k\}$, and let $\mathcal{B}_{k+1}$ be a subspace of the rest of them, $\mathcal{B}_{k+1}=\operatorname{span}\left\{\vec{\gamma}_{i}(t) \mid i=\right.$ $k+1, \ldots, m\}$. If there are $k_{\mathrm{e}}$ positive Lyapunov exponents and UDV does not take place, non-vanishing angles between the expanding subspace $\mathcal{A}_{k_{\mathrm{e}}}$ and the contracting 
one $\mathcal{B}_{k_{\mathrm{e}}+1}$ indicate uniform hyperbolicity. If UDV occurs, some of CLVs either shrink or grow in time, but, nevertheless, the tangent space splitting still can be introduced at arbitrary site $k$. Varying $k$ and checking the angles between corresponding subspaces, we can study the structure of the tangent space in this case.

The presence of UDV automatically breaks the conditions of uniform hyperbolicity, since an invariant splitting of the tangent space into uniformly expanding and contracting subspaces can not exist. But, in principle, the UDV does not exclude a tangent space splitting into subspaces with strictly different rates of expansion or contraction. An $m$-dimensional attractor with UDV can have $k$-dimensional strictly expanding subspace, and $(m-k)$-dimensional subspace whose vectors can either decay or grow but always strictly slower than in the first subspace. In this case angles between vectors from these subspaces will be strictly non-zero. This situation is known without relations to UDV and is referred to as partial hyperbolicity [43]. Partial hyperbolicity does not automatically implies the properties peculiar to uniform hyperbolicity, like, for example, structural stability. However, this is the case provided that some additional requirements are fulfilled [43]. Below we shall check the partial hyperbolicity for our system.

To analyze the possible occurrence of the tangencies between subspaces $\mathcal{A}_{k}$ and $\mathcal{B}_{k+1}$, we have to find two vectors from them having the smallest angle. The angle between these vectors is called the first principal angle [44:

$$
\alpha_{k}=\min \left\{\angle\left(v_{\mathcal{A}}, v_{\mathcal{B}}\right) \mid v_{\mathcal{A}} \in \mathcal{A}_{k}, v_{\mathcal{B}} \in \mathcal{B}_{k+1}\right\}
$$

The tangency corresponds to the vanish of this angle, $\alpha_{k}=0$.

The straightforward way of computing $\alpha_{k}$, as described in [33, includes computation of the whole set of CLVs. But it is much more efficient to consider the angles between $\mathcal{A}_{k}$ and the subspace $\mathcal{B}_{k+1}^{\perp}$ which is the orthogonal complement to $\mathcal{B}_{k+1}$. One have to compute only the first $k$ orthogonal backward and forward Lyapunov vectors and compose the matrix of their scalar products $\mathbf{P}(1: k, 1: k)$. The singular values of this matrix are the cosines of the principal angles between $\mathcal{A}_{k}$ and $\mathcal{B}_{k+1}^{\perp}$. If the smallest one is zero, $\sigma_{\text {min }}=0$, there is a pair of orthogonal vectors from $\mathcal{A}_{k}$ and $\mathcal{B}_{k+1}^{\perp}$. In turn, it means that one can find a collinear pair of vectors from $\mathcal{A}_{k}$ and $\mathcal{B}_{k+1}$, i.e., the tangency occurs. Thus, the smallest principal angle between subspaces $\mathcal{A}_{k}$ and $\mathcal{B}_{k+1}$ can be computed as

$$
\alpha_{k}=\pi / 2-\arccos \left(\sigma_{\min }\right) .
$$

The details of this method can be found in [45].

To detect the occurrences of the tangencies we fix a certain value of the coupling parameter $h$, compute a sufficiently long trajectory of the system (3), find $\alpha_{k}$ at $t=n T$, which corresponds to the consideration of the stroboscopic map, and take the smallest value of the angle. In Fig. 7 this minimal angle is plotted as a function of $h$ for $k=1$, 2 and 3. One can see that in the synchronous regime all three first tangent directions are well isolated from each other. The transition to the non-synchronous regime is accompanied by the sharp drop of the all three angles. Their behavior confirms the 


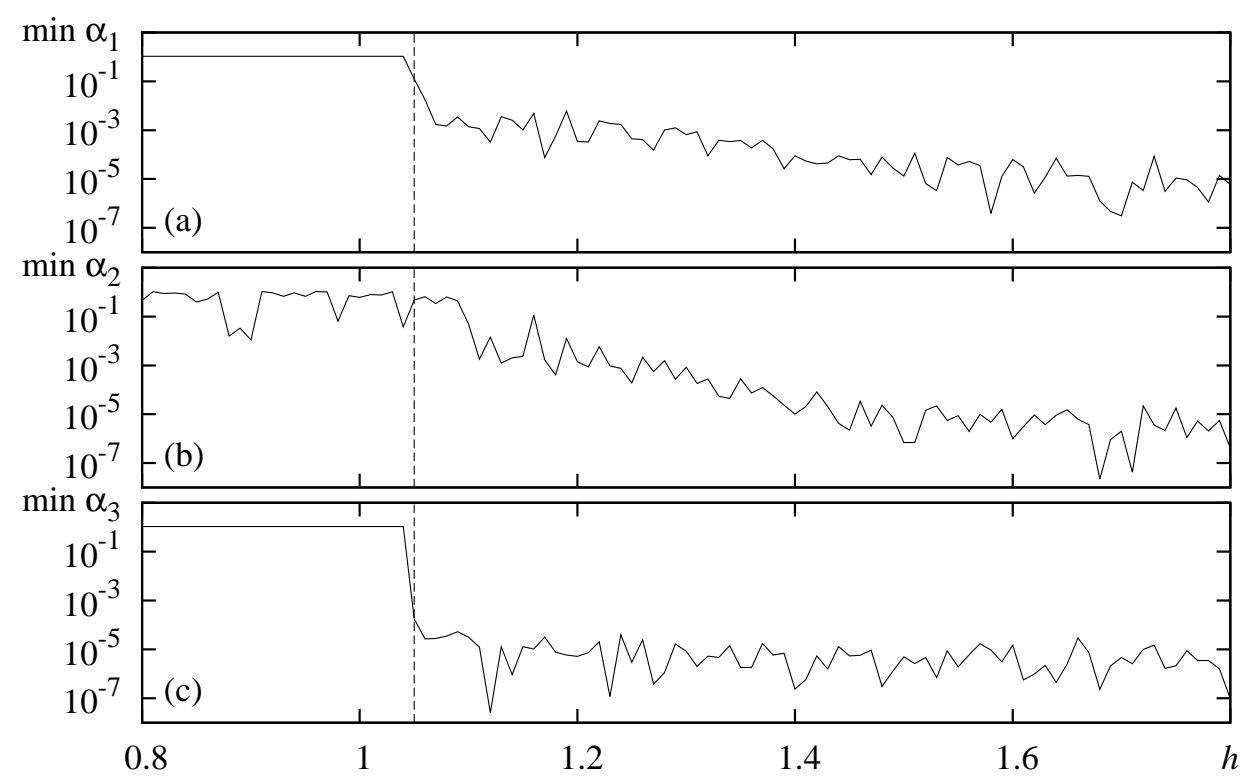

Figure 7. The the smallest angles between tangent subspaces $\mathcal{A}_{k}$ and $\mathcal{B}_{k+1}$ found for $10^{5}$ trajectory points. (a) $k=1$, (b) $k=2$, (c) $k=3$. The vertical line marks the transition to the non-synchronous regime.

expected, due to UDV, violation of the uniform hyperbolicity. Moreover, the drop of $\alpha_{1}$ indicates that the subspaces $\mathcal{A}_{1}$ and $\mathcal{B}_{2}$ have tangencies. It means that an invariant splitting with different expansion rates does not exist, so that even partial hyperbolicity in a strict sense can not be observed above the desynchronization threshold.

Distributions of $\alpha_{k}$ provide a more accurate information about the angles between subspaces. If the distribution is separated well from the origin, then a trajectory never approaches the trajectories with exact tangencies, and one can conjecture that such ones are absent at all. Figure 8(a) illustrates the case of the synchronization at $h=0.8$, when $\mathcal{A}_{1}$ and $\mathcal{B}_{2}$ are the expanding and contracting subspaces, respectively. The distribution of the angle $\alpha_{1}$ between these subspaces is strictly isolated from the origin that confirms the hyperbolicity of the synchronous dynamics.

Figure 8(b) shows the case of UDV at $h=1.2$. The distributions of the angles $\alpha_{1}$, $\alpha_{2}$, and $\alpha_{3}$ indicate the entanglement of the corresponding tangent subspaces. The curve $p\left(\alpha_{1}\right)$ approaches zero being sufficiently small. It means that though the trajectories with the exact tangencies between $\mathcal{A}_{1}$ and $\mathcal{B}_{2}$ exist, they are very rare. The same is the case for the subspaces $\mathcal{A}_{2}$ and $\mathcal{B}_{3}$. But $p\left(\alpha_{3}\right)$ is large at the origin. It means that the most of the tangencies happens around the third covariant Lyapunov vector. Thus, one can conclude that the chaos is non-hyperbolic in this case. However, the rare vanishes of $\alpha_{1}$ and $\alpha_{2}$ indicate that the system almost fulfills the partial hyperbolicity conditions.

Figure 8(c) illustrates the case $h=1.7$ where no Lyapunov exponents fluctuate around zero and there are five unstable directions. The expanding subspace is $\mathcal{A}_{5}$ and the contracting one is $\mathcal{B}_{6}$. The distribution of the angles between these subspaces $p\left(\alpha_{5}\right)$ is high at the origin. Hence, the attractor contains a lot of trajectories with the exact 
a)

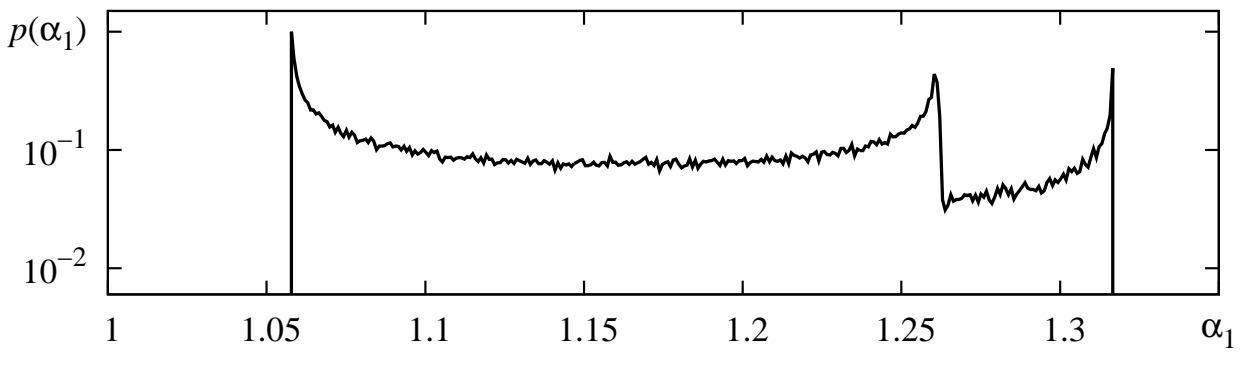

b)

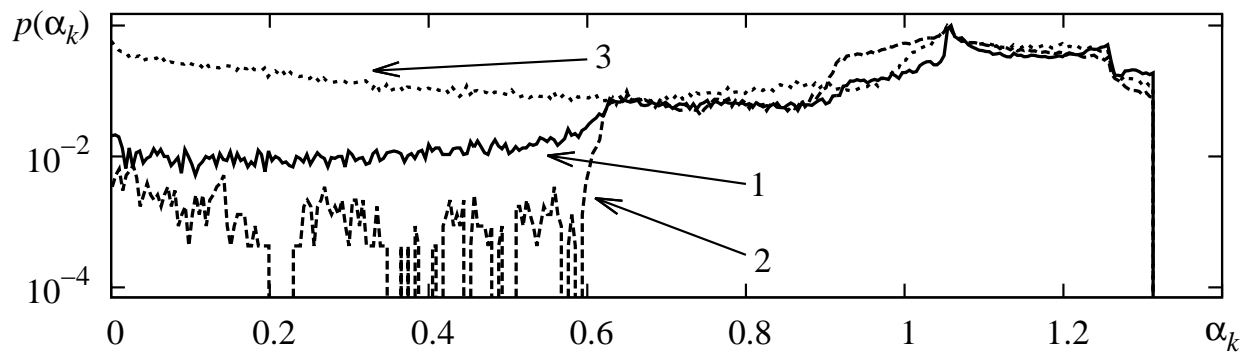

c)

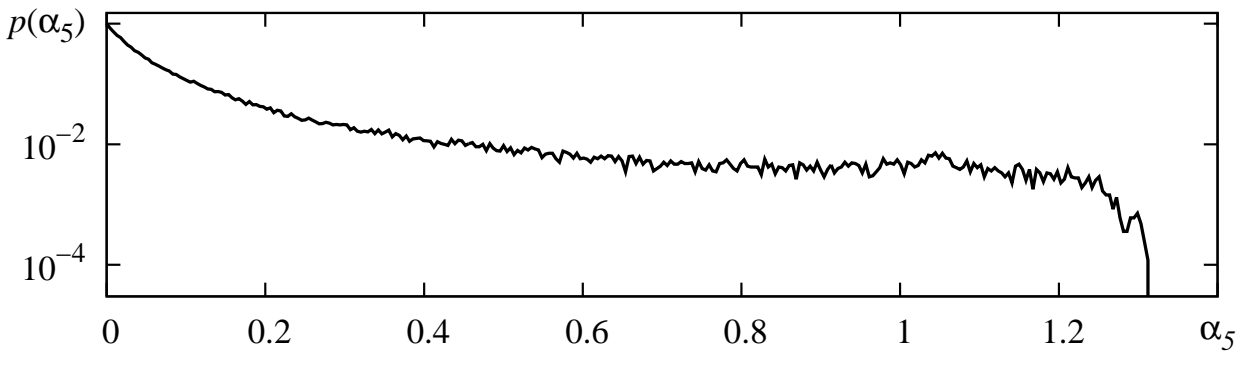

Figure 8. Distributions of the angle $\alpha_{k}$ between subspaces $\mathcal{A}_{k}$ and $\mathcal{B}_{k+1}$ at (a) $h=0.8$, $k=1$, (b) $h=1.2, k=1,2,3$ (arrows 1, 2, 3 respectively), and (c) $h=1.7, k=5$. Each distribution is obtained for $10^{5}$ trajectory points.

tangencies between these subspaces, i.e., it is non-hyperbolic.

To reveal more information about the UDV case, in Fig. 9 we plotted angles $\alpha_{2}$ and $\alpha_{3}$ vs. the distance to the synchronization manifold $\rho$ computed at $10^{5}$ trajectory points. The major balks of points in both cases form bold horizontal stripes. It means that the most probable angles are located around $1 \mathrm{rad}$, and they can be encountered both close to the synchronization manifold, and far from it. In the panel (a) beneath the main stripe there is a series of sparse and hardly visible horizontal stripes. We conjecture that they appear due to the passing of the trajectory close to the embedded periodic orbits. In both panels there are no dense cloud of points near the origin. Zero angles mostly appear at a nonzero $\rho$. For example, in panel (b) one can compare an arch-like structure approaching zero at $\rho \approx 0.2$ and a very dense collection of points around $\alpha_{3}=0, \rho=2$. Hence, the tangencies basically occur when the system goes far from the synchronization manifold. As one can see from Fig. 4(b), since the third FTLE largely fluctuates in this area of the phase space, a strong UDV takes place there. Thus the tangencies in this area happen both at positive and at negative values of this FTLE. Though seldom, $\alpha_{3}$ can nevertheless vanish near the synchronization manifold, see Fig. 9(b). In Fig. 4(b) 


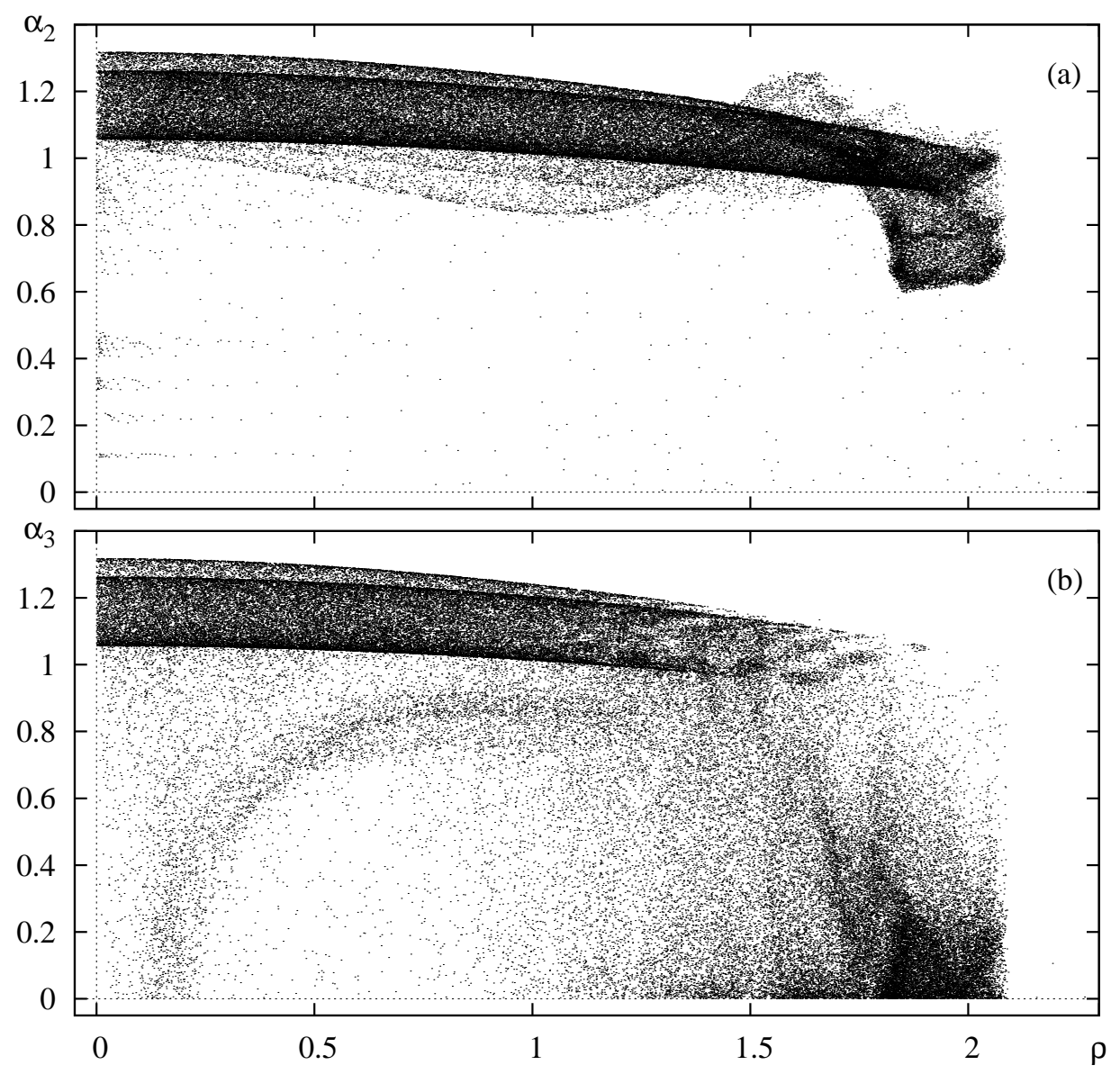

Figure 9. Angle $\alpha_{3}$ and $\alpha_{2}$ vs. transverse distance to the synchronization manifold $\rho$ computed for $10^{5}$ trajectory points. $h=1.2$

in this area the third FTLE can approach zero from above but never become negative. Thus these vanishings of $\alpha_{3}$ indicates the presence of homoclinic tangencies close to the synchronization attractor.

\subsection{Structure of covariant Lyapunov vectors}

The chaos in individual subsystems of the chain (3) appears due to the uniform multiplication of phases, while amplitude dynamics remains rather regular, see section 1 . Thus, it is natural for our system to compute CLVs with respect to the perturbation of phases $\phi$ and amplitudes $r$. Given the Cartesian coordinates $x$ and $y$, one can recompute a perturbation regarding $\phi$ and $r$ as follows: $\tilde{r}=(\partial r / \partial x) \tilde{x}+(\partial r / \partial y) \tilde{y}$, $\tilde{\phi}=(\partial \phi / \partial x) \tilde{x}+(\partial \phi / \partial y) \tilde{y}$

$$
\tilde{\phi}=(-y \tilde{x}+x \tilde{y}) / r^{2}, \quad \tilde{r}=(x \tilde{x}+y \tilde{y}) / r,
$$

where $r=\sqrt{x^{2}+y^{2}}$, and variables with tildes denote elements of CLV, while those without tildes correspond to trajectory coordinates. Thus, given the elements of CLV $\tilde{a}_{n}$ and $\tilde{b}_{n}$, and corresponding trajectory coordinates $a_{n}$ and $b_{n}$, one have to substitute 


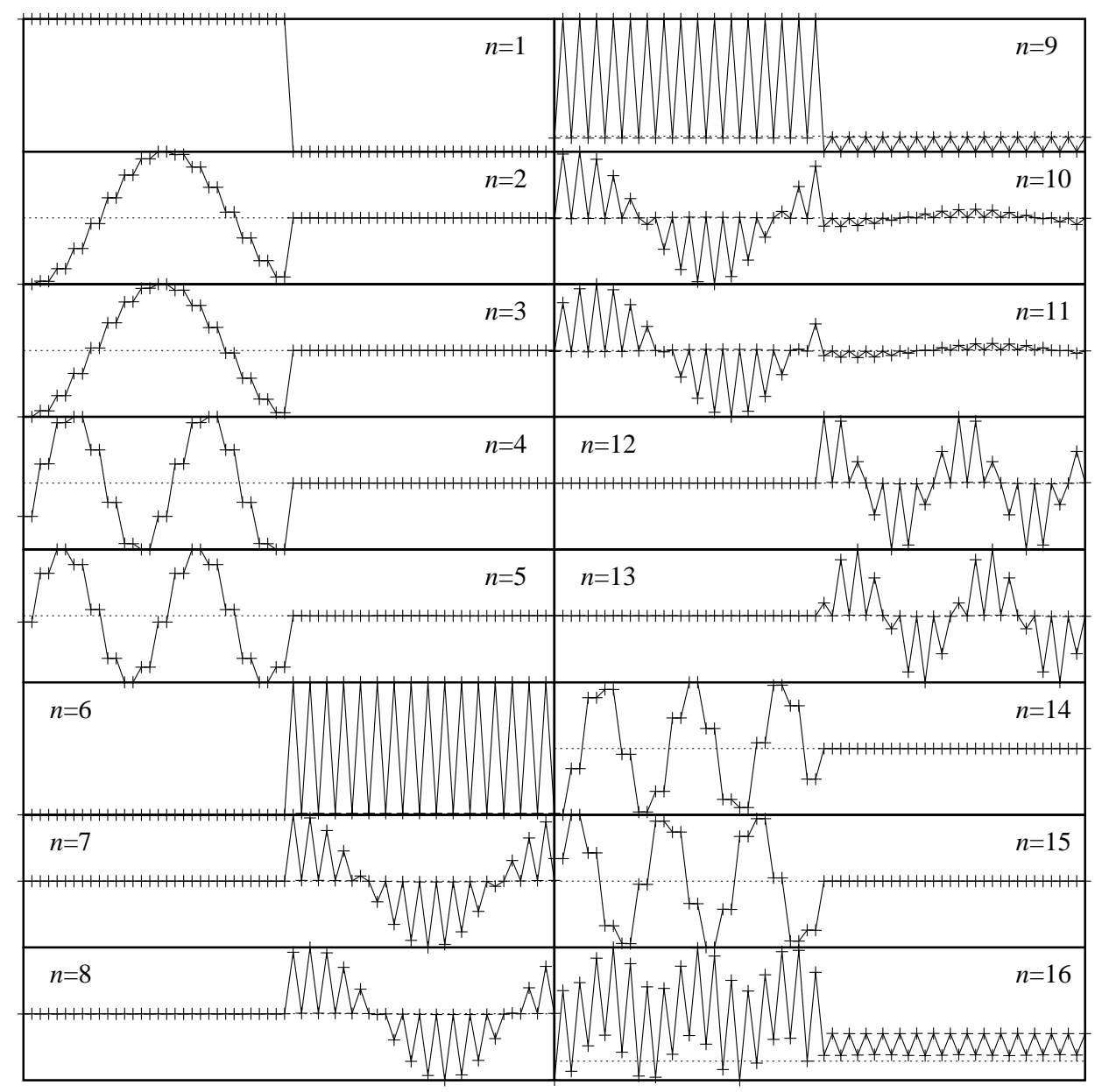

Figure 10. CLVs averaged over $10^{5}$ trajectory points at $h=0.8$. The vector numbers are shown in the panels. The system parameters are as in Fig. 2. In each panel the left 8 points correspond to perturbations of phase variables, and the right 8 ones represent amplitudes. The thin dashed lines show the zero axis

each $\operatorname{Re} a_{n}$ and $\operatorname{Im} a_{n}$ instead of $x$ and $y$, as well as $\operatorname{Re} \tilde{a}_{n}$ and $\operatorname{Im} \tilde{a}_{n}$ instead of $\tilde{x}$ and $\tilde{y}$, and compute corresponding $\tilde{\phi}_{n}, \tilde{r}_{n}$. The same have to be done for $b_{n}$.

To visualize the structure of CLVs we find them for many trajectory points, recompute with respect to phases and amplitudes, normalize and average over the trajectory. The results are shown in Figs. 10, 11, 12, When chaos is hyperbolic and chain elements are synchronized, as in Fig. 10, the CLVs are highly regular. The first one corresponds to the single positive Lyapunov exponent. We observe that, as expected, the expanding perturbation grows strictly along phase variables. Notice high homogeneity of the vector. Phases in each site of the vector grow identically which is a manifestation of the synchronous regime. All other CLVs show the contracting directions. Again the phase and amplitude perturbations are isolated. The first four contracting vectors $n=2,3,4$, and 5 represent the phase perturbation, the next three ones, $n=6,7$, and 8 are the amplitude ones, and so on. Vectors $n=9,10,11,16$ represent weakly mixed case: together with the phase perturbation there is some perturbation along amplitude 


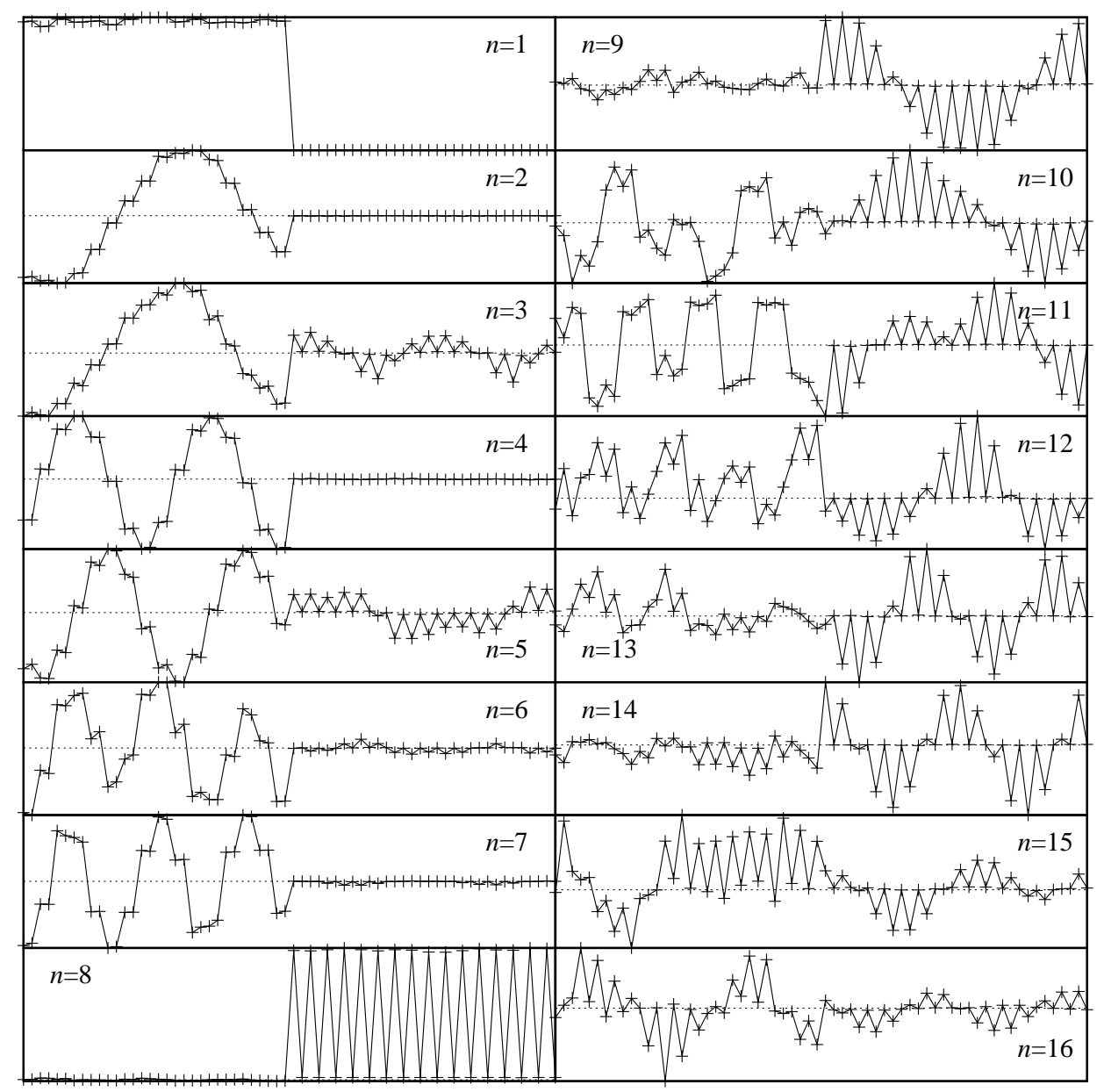

Figure 11. Averaged CLVs for $h=1.2$.

directions. But these additions are small so that the phase directions dominate anyway.

The UDV case is illustrated in Fig. 11. One can see that three first vectors are basically directed along the phase subspace, i.e., the phases undergo the expansion. Observe high similarity of the first five vectors with the previous case. After the transition to the non-synchronous regime the structure of the expanding vector $n=1$ remains almost unaltered, while the vectors 3 and 5 are rotated a little towards the amplitude directions. The rotation of the third vector is in charge of the emergence of entanglement of the expanding and contracting subspaces in this case that is discussed in section 3.4. Two new phase perturbation vectors $n=6$ and 7 appear that are absent in the synchronization regime in Fig. 10, The subsequent amplitude perturbation vectors $n=8,9$, and 10 correspond to the vectors $n=6,7$, and 8 in Fig. 10, Further correspondence between CLVs in synchronous and UDV cases are rather untraceable by a visual inspection.

Figure 12 illustrates the "common" chaos observed beyond hyperbolic and UDV cases. There are five positive Lyapunov exponents and again, as before, all corresponding vectors are basically represent the phase perturbation. The similarity of the first seven 


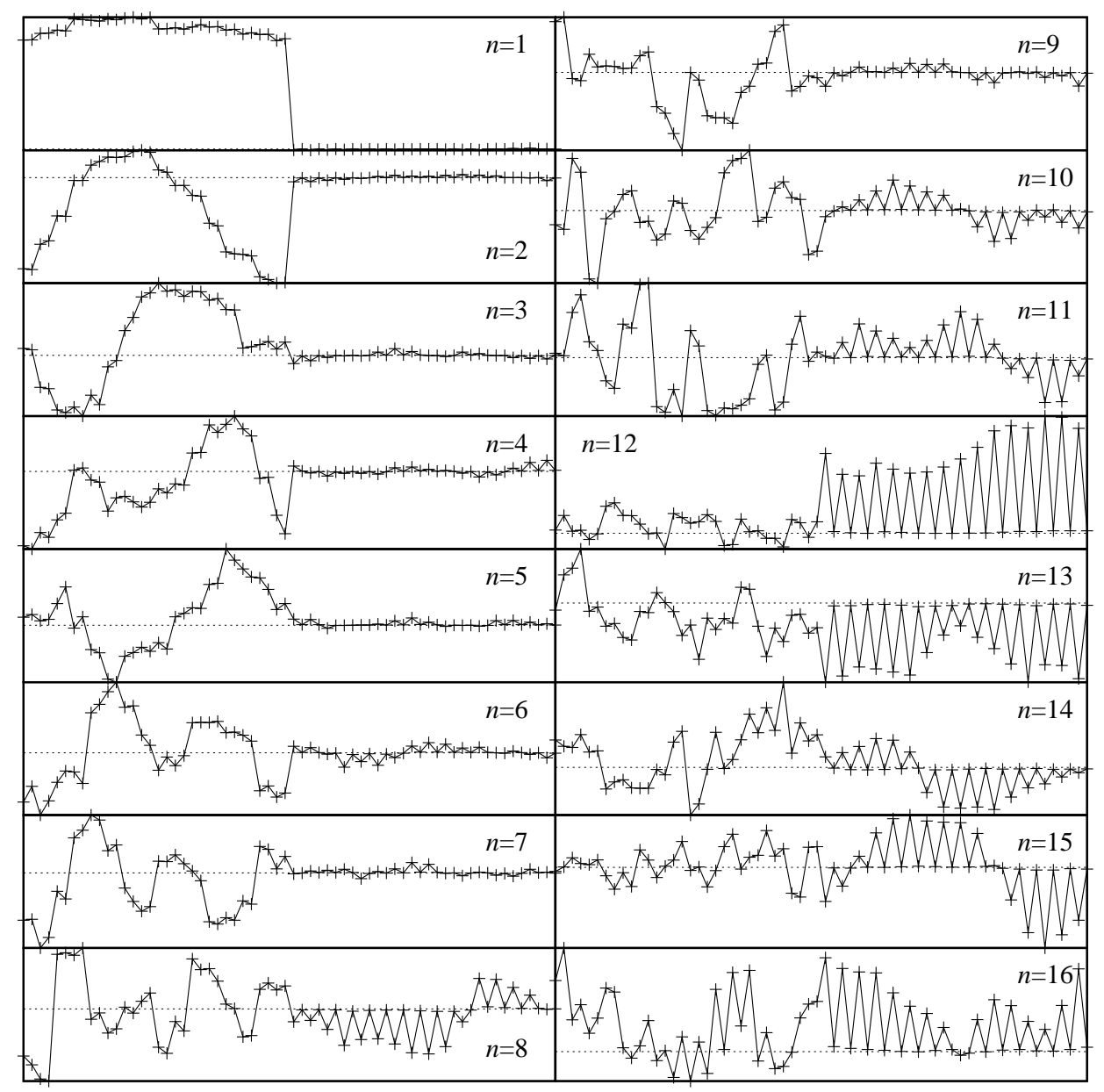

Figure 12. Averaged CLVs for $h=1.7$.

vectors with the previous cases is still noticeable. The first vectors consists of the homogeneous phase and zero amplitude parts, i.e., the strongest expansion occurs along the diagonal in the phase perturbation subspace. The vectors with indexes running from $n=2$ to 7 has more of less similar structure with the corresponding vectors in the UDV case. Perhaps, the subsequent four vectors $n=8,9,10$, and 11 can be treated as new phase perturbation vectors, while the vector $n=12$ corresponds to the vector $n=8$ in Fig. 11 and $n=6$ in Fig. 10 .

In principle, the isolation of phase and amplitude perturbations, i.e., small amplitude or phase components of the averaged vectors, could be obtained if their fluctuations along the trajectory were high and uniformly distributed. But this is not the case for our system. The fluctuations of the phase and amplitude components are sufficiently small. To test it, we have computed in the same way CLVs averaged over just 10 trajectory points. The vectors for synchronous hyperbolic case at $h=0.8$ look almost indistinguishable from those shown in Fig. 10 up to a shift of maxima obviously explained by the periodic boundaries conditions. For two other case the coincidence is not so perfect but is still very good. Figure 13 shows the most different vectors: 


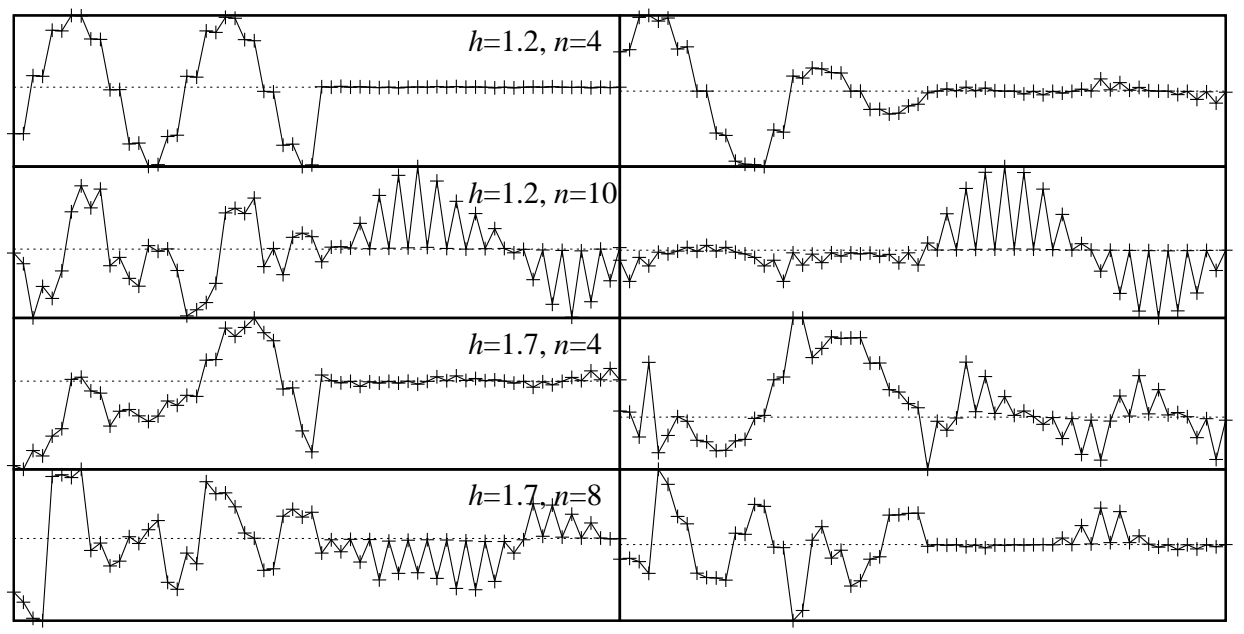

Figure 13. CLVs averaged over $10^{5}$ trajectory points (the left column of panels) and over 10 points (the right column)

the left columns reproduces the vectors from Figs. 11 and 12 and the right one shows the corresponding vectors with the poor averaging. One sees that these vectors are qualitatively similar.

\section{Summary and discussion}

We discussed the violation of the synchronous uniformly hyperbolic chaos in a chain of diffusively coupled hyperbolic chaotic oscillators. The most important feature of these oscillators is that they admit a physical implementation, so that our results can be tested experimentally.

We observed the following scenario. The breakdown of the synchronization begins when the second and the third Lyapunov exponents simultaneously approach zero as the coupling strength decays. The second FTLE starts to fluctuate, however the sign changings are rare, and the range of coupling where the fluctuations occur is very narrow. Within this range the on-off intermittency is observed induced by UDV. In turn, the UDV inside this range arises due to the invariant orbits embedded into the synchronization attractor. Each of these orbits loses its transverse stability at certain coupling strength, so that the attractor contains subsets with various dimensions of unstable and stable manifolds. As the coupling decays further, the second FTLE leaves the vicinity of zero, while the third FTLE fluctuates changing its sign. This happens within a very wide range of the coupling parameter. These fluctuations are related to the UDV arising due to so called emergent set. This is the set of invariant orbits with various dimensions of unstable and stable manifolds. These orbits are located far from the synchronization manifold and embedded into the non-synchronous attractor. The Gaussian approximation of probability density of fluctuations of unstable dimension was analyzed, and the slow decay of the probability, compared to non-UDV cases, was revealed. 
The test of angles between tangent subspaces confirms the expected violation of the uniform hyperbolicity in the UDV case. Moreover, the tests show even the absence of partial hyperbolicity, which is not prohibited in UDV regime. However, the vanishings of angles responsible for this occur very seldom, so that our system in the UDV regime is almost partially hyperbolic. The partial hyperbolicity does not automatically implies the properties peculiar for the uniform hyperbolicity. Further study is required to understand how exactly this almost partial hyperbolicity manifests itself.

Typically, CLVs strongly oscillate changing their directions from point to point. However, for our system, being considered regarded to phases and amplitudes of partial oscillators, the CLVs remain almost constant. Thus, we are able to discuss the transformation of their structure with the decay of the coupling strength. The visual inspection reveals the isolation of phase and amplitude perturbations. This basically occurs for first vectors, while the vectors with high numbers contains both phase and amplitude components.

\section{Acknowledgments}

The research is supported by RFBR-DFG grant 11-02-91334.

\section{References}

[1] A. A. Andronov, A. A. Vitt, and S. E. Khaikin. Theory of Oscillators. Dover Publications, 2 edition, 2011.

[2] L. Shilnikov. Mathematical problems of nonlinear dynamics: A tutorial. Int. J. of Bifurcation and Chaos, 7:1353-2001, 1997.

[3] D. V. Anosov et al. Dynamical Systems IX: Dynamical Systems with Hyperbolic Behaviour, volume 9 of Encyclopaedia of Mathematical Sciences. Springer, New York, 1995.

[4] S. Smale. Differentiable dynamical systems. Bull. Amer. Math. Soc., 73(2):747-817, 1967.

[5] R. F. Williams. Expanding attractors. Publ. math. de l'I.H.É.S., 43:169-203, 1974.

[6] R. V. Plykin. Sources and sinks of a-diffeomorphisms of surfaces. Math. USSR Sb., 23(2):233-253, 1974.

[7] S. P. Kuznetsov. Example of a physical system with a hyperbolic attractor of the Smale-Williams type. Phys. Rev Lett., 95:144101, 2005.

[8] S. P. Kuznetsov and E. P. Seleznev. A strange attractor of the smale-williams type in the chaotic dynamics of a physical system. JETP, 102(2):355-364, 2006.

[9] S. P. Kuznetsov. Hyperbolic Chaos: A Physicist's View. Higher Education Press in Springer, Beijing/Berlin, 2012.

[10] S. P. Kuznetsov. Dynamical chaos and uniformly hyperbolic attractors: from mathematics to physics. Physics-Uspekhi, 54(2):119-144, 2011.

[11] A. Katok and B. Hasselblatt. Introduction to the modern theory of dynamical systems. Cambridge university press, 1995.

[12] Eric J. Kostelich, Ittai Kan, Celso Grebogi, Edward Ott, and James A. Yorke. Unstable dimension variability: A source of nonhyperbolicity in chaotic systems. Physica D, 109(1-2):81-90, 1997.

[13] RF Pereira, SE de S Pinto, RL Viana, SR Lopes, and C. Grebogi. Periodic orbit analysis at the onset of the unstable dimension variability and at the blowout bifurcation. Chaos, 17(2):3131, 2007. 
[14] Tim Sauer, Celso Grebogi, and James A. Yorke. How long do numerical chaotic solutions remain valid? Phys. Rev. Lett., 79:59-62, Jul 1997.

[15] Timothy D. Sauer. Shadowing breakdown and large errors in dynamical simulations of physical systems. Phys. Rev. E, 65:036220, Feb 2002.

[16] D. V. Anosov. Geodesic flows on closed riemannian manifolds of negative curvature. Proc. Steklov Inst. Math., 90:1-235, 1967.

[17] R. Bowen. $\omega$-limit sets for axiom a diffeomorphisms. Journal of differential equations, 18(2):333339, 1975.

[18] Celso Grebogi, Stephen M. Hammel, James A. Yorke, and Tim Sauer. Shadowing of physical trajectories in chaotic dynamics: Containment and refinement. Phys. Rev. Lett., 65:1527-1530, Sep 1990.

[19] R. F. Pereira, S. Camargo, S. E. de S. Pinto, S. R. Lopes, and R. L. Viana. Periodic-orbit analysis and scaling laws of intermingled basins of attraction in an ecological dynamical system. Phys. Rev. E, 78:056214, Nov 2008.

[20] Filipe J. Romeiras, Celso Grebogi, Edward Ott, and W.P. Dayawansa. Controlling chaotic dynamical systems. Physica D: Nonlinear Phenomena, 58(1-4):165-192, 1992.

[21] Ying-Cheng Lai, David Lerner, Kaj Williams, and Celso Grebogi. Unstable dimension variability in coupled chaotic systems. Phys. Rev. E, 60:5445-5454, Nov 1999.

[22] Ricardo L. Viana and Celso Grebogi. Unstable dimension variability and synchronization of chaotic systems. Phys. Rev. E, 62:462-468, Jul 2000.

[23] Ernest Barreto and Paul So. Mechanisms for the development of unstable dimension variability and the breakdown of shadowing in coupled chaotic systems. Phys. Rev. Lett., 85:2490-2493, Sep 2000.

[24] N. Platt, E. A. Spiegel, and C. Tresser. On-off intermittency: a mechanism for bursting. Phys. Rev. Lett., 70:279-282, 1993.

[25] A. Pikovsky, M. Rosenblum, and J. Kurths. Synchronization: A Universal Concept in Nonlinear Sciences. Cambridge Nonlinear Science Series. Cambridge University Press, 2003.

[26] Edward Ott and John C. Sommerer. Blowout bifurcations: the occurrence of riddled basins and on-off intermittency. Physics Letters A, 188(1):39 - 47, 1994.

[27] F. Ginelli, P. Poggi, A. Turchi, H. Chaté, R. Livi, and A. Politi. Characterizing dynamics with covariant Lyapunov vectors. Phys. Rev. Lett., 99:130601, 2007.

[28] C. L. Wolfe and R. M. Samelson. An efficient method for recovering Lyapunov vectors from singular vectors. Tellus A, 59A:355-366, 2007.

[29] Pavel Kuptsov and Ulrich Parlitz. Theory and computation of covariant Lyapunov vectors. Journal of Nonlinear Science, 22(5):727-762, 2012.

[30] F. Ginelli, H. Chate, R. Livi, and A. Politi. Covariant Lyapunov vectors: a practical guide. J Phys A, Special issue on Lyapunov analysis, 2013.

[31] L. A. Bunimovich and Ya. G. Sinai. Spacetime chaos in coupled map lattices. Nonlinearity, 1:491-516, 1988.

[32] L. A. Bunimovich and Ya. G. Sinai. Statistical mechanics of coupled map lattices. In Theory and application of coupled map lattices, pages 169-189. Wiley, New York, 1993.

[33] Pavel V. Kuptsov and Sergey P. Kuznetsov. Violation of hyperbolicity in a diffusive medium with local hyperbolic attractor. Physical Review E, 80(1):016205, 2009.

[34] Pavel V. Kuptsov, Sergey P. Kuznetsov, and Arkady Pikovsky. Hyperbolic chaos of Turing patterns. Phys. Rev. Lett., 108:194101, May 2012.

[35] R. L. Viana, C. Grebogi, S. E. de S. Pinto, S. R. Lopes, A. M. Batista, and J. Kurths. Validity of numerical trajectories in the synchronization transition of complex systems. Phys. Rev. E, 68:067204, Dec 2003.

[36] D. Wilczak. Uniformly hyperbolic attractor of the Smale-Williams type for a Poincaré map in the Kuznetsov system. SIAM J. Appl. Dyn. Syst., 9:1263-1283, 2010.

[37] Pavel V. Kuptsov and Sergey P. Kuznetsov. Synchronization and collective behavior of a coupled 
map lattice with unidirectional coupling and periodic boundary conditions. Prikl. Nelin. Dinam. (Appl. Nonlin. Dynam.), 12(3):3-22, 2004. in Russian.

[38] S. E. de S. Pinto, I. L. Caldas, A. M. Batista, S. R. Lopes, and R. L. Viana. Transversal dynamics of a non-locally-coupled map lattice. Phys. Rev. E, 76:017202, Jul 2007.

[39] Xingang Wang, Shuguang Guan, Ying-Cheng Lai, Baowen Li, and Choy Heng Lai. Desynchronization and on-off intermittency in complex networks. EPL (Europhysics Letters), 88(2):28001, 2009.

[40] Sergey P. Kuznetsov Pavel V. Kuptsov. Transition to a synchronous chaos regime in a system of coupled non-autonomous oscillators presented in terms of amplitude equations. Rus. J. Nonlin. Dyn., 2(3):307-331, 2006.

[41] Pavel V. Kuptsov and Antonio Politi. Large-deviation approach to space-time chaos. Phys. Rev. Lett., 107:114101, Sep 2011.

[42] R. Chandramouli and N. Ranganathan. Computing the bivariate gaussian probability integral. Signal Processing Letters, IEEE, 6:129-131, 1999.

[43] Ya. B. Pesin. Lectures on partial hyperbolicity and stable ergodicity. European Mathematical Society Publishing House, Zürich, 2004.

[44] G. H. Golub and C. F. van Loan. Matrix computations. The Johns Hopkins University Press, Baltimore, 3rd edition, 1996.

[45] Pavel V. Kuptsov. Fast numerical test of hyperbolic chaos. Phys. Rev. E, 85:015203, Jan 2012. 